\title{
Electric charge quantization and the muon anomalous magnetic moment
}

\author{
C. A. de S. Pires* and P. S. Rodrigues da Silva ${ }^{\dagger}$ \\ Instituto de Física Teórica, Universidade Estadual Paulista, Rua Pamplona 145, 01405-900 São Paulo, São Paulo, Brazil
}

(Received 3 September 2001; published 5 April 2002)

\begin{abstract}
We investigate some proposals to solve the electric charge quantization puzzle that simultaneously explain the recent measured deviation on the muon anomalous magnetic moment. For this we assess extensions of the electro-weak standard model spanning modifications on the scalar sector only. It is interesting to verify that one can have modest extensions which easily account for the solution for both problems.
\end{abstract}

DOI: 10.1103/PhysRevD.65.076011

PACS number(s): $12.90 .+\mathrm{b}, 12.60 . \mathrm{Fr}$

\section{INTRODUCTION}

It is known that the minimal standard model (MSM), though very well tested at the experimental level, is not the most complete theory of particle physics since some important questions cannot be explained without quoting physics beyond its minimal structure. Among these questions, there is an intriguing one which concerns the observation of, to an extremely high accuracy, exact electric charge quantization (ECQ). The fact that differences among electric charges of known particles are given in terms of integer numbers implies that any reasonable theory for elementary particles has to accommodate such a quantization or, at least, must give a strong reason for the violation to be so small.

In the beginning of the 1990's, the question concerning ECQ was studied inside the MSM through classical and quantum constraints [1-8]. The classical constraints come from imposing the invariance of the Lagrangian under the standard gauge group transformation, $\mathrm{SU}(3)_{C} \otimes \mathrm{SU}(2)_{L}$ $\otimes \mathrm{U}(1)_{Y}$, while the quantum constraints are a consequence of anomaly cancellation via the computation of the triangle diagrams [9]. If these two constraints fix the pattern of quantization of hypercharge, they automatically establish the pattern of quantization for the fermion electric charge through the formula

$$
Q=T^{3}+\frac{Y}{2}
$$

where $T^{3}$ is the diagonal generator of $\mathrm{SU}(2)$ and $Y$ is the hypercharge of the particle with charge $Q$. All sectors of the MSM Lagrangian are trivially gauge-invariant by the standard gauge symmetry, except the Yukawa sector. It is enough to focus only on this part of the Lagrangian wherein the imposition of gauge invariance implies the useful classical relations among the hypercharges of fermions and scalars, still arbitrary at this level. This formalism can be extended to any model based on a semisimple group with the structure $\mathrm{SU}(3)_{C} \otimes \mathrm{SU}(n)_{L} \otimes \mathrm{U}(1)_{X}$. In this case, the constraints must fall over the quantum number $X$, and the electric charge is

\footnotetext{
*Electronic address: cpires@ift.unesp.br

${ }^{\dagger}$ Electronic address: fedel@ift.unesp.br
}

given by a combination of $X$ and the diagonal generators, $T_{d}$, of $\operatorname{SU}(n)$. Then, instead of Eq. (1) we get

$$
Q=\sum T_{d}+\frac{X}{2}
$$

It is interesting to recall that the MSM with only one family has enough content to provide ECQ [1,2]. However, when three families are considered, this property is lost and the theory undergoes an effect known as electric charge dequantization. The reason is that with three families, combinations of additional $U(1)$ gaugeable symmetries start to plague the model in the sense that, besides hypercharge, one has now a continuous amount of extra quantum numbers. These cannot be fixed by the present constraints on the MSM, forbidding ECQ. Then, we can state that if the formalism is applied to extensions of the MSM and predicts the ECQ, this means the model has no other global symmetry that can be promoted to local symmetry besides $\mathrm{U}(1)_{Y}[1,2]$. Conversely, if there exists some global symmetry, potentially "gaugeable," usually called hidden symmetry, we cannot obtain enough classical or quantum constraints to have ECQ. Unfortunately, this is the case if one sticks to the MSM alone $[1-3,8]$, where there exist three hidden symmetries (when mixed gauge-gravitational anomalies are also considered):

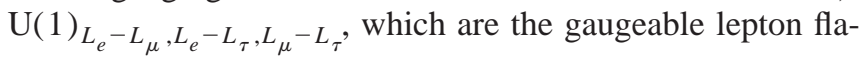
vor symmetries. This implies that electric charge in the MSM cannot be quantized and has the following expression: $Q$ $=Q_{\mathrm{st}}+\epsilon\left(L_{e}-L_{\mu}\right.$ or $L_{e}-L_{\tau}$ or $\left.L_{\mu}-L_{\tau}\right) \quad[1,2]$, where $Q_{\mathrm{st}}$ is the standard assigned charge to the respective particle and $\epsilon$ is an arbitrary continuous parameter.

This approach elucidates the method one needs to follow in order to suitably construct models beyond the MSM to naturally obtain ECQ: extensions of the MSM that attempt to predict ECQ must explicitly break any hidden symmetry at the Lagrangian level. In this direction, various extensions of the MSM were analyzed in the literature $[1,3,4,6,8]$. The main conclusion arrived at in those works was that if neutrinos are massive and Majorana-like, then they automatically yield ECQ [4]. This is easy to understand since Majorana mass terms do require violation of the lepton number and, as a consequence, the hidden symmetries

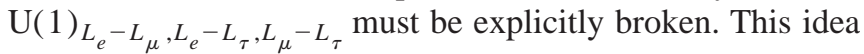
received a great deal of attention at that time because there was experimental evidence of neutrino oscillations, whose solution requires that neutrinos be massive. Recently, such 
oscillations were confirmed, however it is not possible to infer the true nature of neutrinos from those experiments, whether Majorana or Dirac, leaving the ECQ puzzle unsolved [10].

Nevertheless, a new measurement of the anomalous magnetic moment of the muon, $(g-2)_{\mu}$, has shown a deviation from the theoretical result [11], pointing to possible new physics beyond the MSM. Since the deviation is in the leptonic sector, it turns out that its solution can, conveniently, be cast in such a way to simultaneously solve the ECQ puzzle. This is what we propose in this work. In order to do that, we first review the casting of ECQ in the MSM at Sec. II. Then we present, at Sec. III, simple MSM extensions suitable to correctly get the ECQ and show, in Sec. IV, that some of these proposals can explain the $(g-2)_{\mu}$ deviation. Finally, we present our conclusions in Sec. V.

\section{ECQ WITHIN THE STANDARD MODEL}

In order to better understand the method employed to study the ECQ, we start by reviewing the procedure in the context of the MSM. The MSM is defined by the gauge structure $\mathrm{SU}(3)_{C} \otimes \mathrm{SU}(2)_{L} \otimes \mathrm{U}(1)_{Y}$. According to this structure, let us attribute the following representation to the fermions:

$$
\begin{aligned}
& L_{i_{L}}=\left(\begin{array}{l}
\nu \\
e
\end{array}\right)_{L} \sim\left(\mathbf{1}, 2, Y_{i_{L}}\right), \quad l_{i_{R}} \sim\left(\mathbf{1}, \mathbf{1}, Y_{i_{l}}\right), \\
& Q_{i_{L}}=\left(\begin{array}{l}
u \\
d
\end{array}\right)_{L} \sim\left(\mathbf{3}, 2, Y_{i_{Q}}\right), \\
& u_{i_{R}} \sim\left(\mathbf{3}, \mathbf{1}, Y_{i_{u}}\right), \quad d_{i_{R}} \sim\left(\mathbf{3}, \mathbf{1}, Y_{i_{d}}\right),
\end{aligned}
$$

where the index $i=1,2,3$ labels the three different families of leptons, $L_{i_{L}}$ and $l_{i_{R}}$, and quarks, $Q_{i_{L}}, u_{i_{R}}$, and $d_{i_{R}}$. The subindices $L$ and $R$ stand for left-handed and right-handed projections. The Yukawa sector of the MSM is given by

$$
\mathcal{L}^{Y}=g_{i i}^{l} \bar{L}_{i_{L}} \phi l_{i_{R}}+g_{i j}^{u} \bar{Q}_{i_{L}} \widetilde{\phi} u_{j_{R}}+g_{i j}^{d} \bar{Q}_{i_{L}} \phi d_{j_{R}}+\text { H.c. },
$$

with $g_{i i}^{l}, g_{i j}^{u}$, and $g_{i j}^{d}$ [3] the usual Yukawa couplings. Under the $\mathrm{U}(1)_{Y}$ gauge invariance, Eq. (4) gives us the following constraints:

$$
Y_{i_{l}}=Y_{i_{L}}-1, \quad Y_{j_{u}}=Y_{i_{Q}}+1, \quad Y_{j_{d}}=Y_{i_{Q}}-1 .
$$

The last two constraints amount to

$$
\begin{aligned}
& Y_{1_{u}}=Y_{2_{u}}=Y_{3_{u}}=Y_{u}, \\
& Y_{1_{d}}=Y_{2_{d}}=Y_{3_{d}}=Y_{d}, \\
& Y_{1_{Q}}=Y_{2_{Q}}=Y_{3_{Q}}=Y_{Q},
\end{aligned}
$$

leaving us with the true constraints

$$
Y_{i_{l}}=Y_{i_{L}}-1, \quad Y_{u}=Y_{Q}+1, \quad Y_{d}=Y_{Q}-1 .
$$

It is clear from the equations above that we have four free parameters. In order to fix these parameters, we need additional equations which can be taken from the anomaly cancellation constraints. However, once Eq. (7) is taken into account, the MSM presents only two nontrivial anomalies whose vanishing condition furnishes two more constraints over the hypercharges,

$$
\begin{aligned}
{\left[\mathrm{SU}(2)_{L}\right]^{2} \mathrm{U}(1)_{Y} } & \Rightarrow 9 Y_{Q}+\sum_{i}^{3} Y_{L_{i}}=0, \\
{\left[\mathrm{U}(1)_{Y}\right]^{3} } & \Rightarrow 18 Y_{Q}^{3}-9 Y_{u}^{3}-9 Y_{d}^{3}+\sum_{i}^{3}\left(2 Y_{i_{L}}^{3}-Y_{i_{l}}^{3}\right) \\
& =0,
\end{aligned}
$$

and these, together with Eq. (7), are not enough to fix all the hypercharges. In short, this is so because the leptonic sector is not as constrained as the quark sector, and presents extra global symmetries which can be promoted to gauge symmetries, namely $\mathrm{U}(1)_{L_{e}-L_{\mu}, L_{e}-L_{\tau}, L_{\mu}-L_{\tau}}$. As was remarked in the Introduction, the presence of these gaugeable symmetries precludes ECQ for the MSM.

In view of this, we can say that the MSM lacks additional constraints in the leptonic sector once ECQ is realized in nature. If one focuses on this issue only, it is clear that appropriate extensions of the MSM have to be related mainly to the leptonic sector. Moreover, they should include terms that explicitly forbid the above hidden symmetries, automatically reducing the number of free leptonic hypercharges to only one, i.e., the new terms ought to provide the following relations among such hypercharges:

$$
\left.\begin{array}{c}
Y_{1_{L}}=Y_{2_{L}}=Y_{3_{L}}=Y_{L} \\
Y_{1_{l}}=Y_{2_{l}}=Y_{3_{l}}=Y_{l}
\end{array}\right\} \Rightarrow Y_{l}=Y_{L}-1 .
$$

Substituting this result in Eq. (7) yields

$$
Y_{l}=Y_{L}-1, \quad Y_{u}=Y_{Q}+1, \quad Y_{d}=Y_{Q}-1,
$$

which, along with the anomaly cancellation constraints in Eq. (8), are enough to fix the hypercharges,

$$
\begin{aligned}
& Y_{L}=-1, Y_{l}=-2, Y_{Q}=1 / 3, \\
& Y_{u}=4 / 3, \quad Y_{d}=-2 / 3 .
\end{aligned}
$$

This leads automatically to the ECQ with the correct electric charge pattern, $Q_{l}=-1, Q_{u}=1 / 3, Q_{d}=-2 / 3$.

Observe that we can be driven to the relations in Eq. (9) from operators involving bilinear fermionic products like $\bar{\Psi}_{i}^{c} \Psi_{j} .{ }^{1}$ These operators violate twice the total fermion num-

\footnotetext{
${ }^{1}$ It is opportune to remark that including Dirac massive neutrinos would not lead to a solution for the ECQ problem, although it would lead to classical constraints for the leptons as those obtained for the quarks in Eq. (7). This is so because now the anomaly constraints in Eq. (8) would not be independent, and another rela-
} 
ber, and the simplest kinds of particles that can couple to this sort of bilinear, embedded in a small extension of the MSM, are scalars carrying $F=-2$. Vector bosons could also play this role, but would demand an enlargement of the MSM symmetry. We have chosen to adopt the simple scalar picture, and it is in this direction that we develop the remainder of this work.

\section{ECQ BEYOND STANDARD MODEL}

Guided by the procedure employed in Sec. II, we will investigate appropriate MSM extensions in light of ECQ. Some attempts were already considered where new neutral fermion singlets [6] or a second Higgs doublet are added to the MSM [3]. A catalogue of baryon number violating scalar interactions was also considered in Ref. [12]. However, it is interesting to remark that MSM modifications in the direction of eliminating the hidden symmetries, $\mathrm{U}(1)_{L_{e}-L_{\mu}, L_{e}-L_{\tau}, L_{\mu}-L_{\tau}}$, by performing simple additions in its scalar sector, such as the inclusion of singlet scalars, singly and doubly charged, were not considered yet. Of course, these scalars allow some nonstandard leptonic interactions and there was little experimental motivation for such an endeavor, except for neutrino physics. In view of the new experimental results related to the $(g-2)_{\mu}$, we are going to limit our study of ECQ to extensions which modify only the scalar sector and could equally offer an explanation for the discrepancy between the theoretical and experimental results on $(g-2)_{\mu}[11]$.

As briefly pointed out in the end of the last section, if one wishes to break the lepton flavor symmetry combinations,

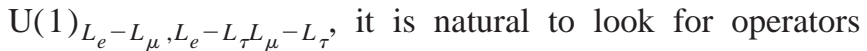
composed of fermions and scalars involving the bilinear product, $\bar{\Psi}_{i}^{c} \Psi_{j}$, which properly accommodates family number violation interactions. Here $\Psi$ is a fermion in a doublet or singlet representation, the indices $i$ and $j$ denote the family, and the superscript $c$ means charge conjugation. In what follows, we consider two kinds of interaction among fermions demanding different species of scalars.

\section{A. Lepton-lepton interactions}

Within the fermionic representation content of the MSM, the possible bilinear products involving leptons only are

$$
\begin{gathered}
\overline{\left(L_{i_{L}}\right)^{c}} L_{j_{L}} \sim\left[\mathbf{1}, \mathbf{1} \oplus \mathbf{3},-\left(Y_{i_{L}}+Y_{j_{L}}\right)\right], \\
\overline{\left(l_{i_{R}}\right)^{c}} l_{j_{R}} \sim\left[\mathbf{1}, \mathbf{1},-\left(Y_{i_{l}}+Y_{j_{l}}\right)\right] .
\end{gathered}
$$

The first term in Eq. (11) requires either a singlet or a triplet of scalars, both carrying a net total lepton number, $L$ $=-2$. Let us first analyze the case of a singlet. With a scalar singlet $h \sim\left(\mathbf{1}, \mathbf{1}, Y_{h}\right)$, we can write the following Yukawa interaction:

tion would be required to fix all the hypercharges of the model.

$$
\mathcal{L}_{h}^{Y}=f_{i j} \overline{\left(L_{i_{L}}\right)^{c}} L_{j_{L}} h+\text { H.c., }
$$

where $f_{i j}$ is a component of an antisymmetric $(3 \times 3)$ family mixing matrix. The above Lagrangian gives us the classical constraint relation among the hypercharges,

$$
Y_{i_{L}}+Y_{j_{L}}+Y_{h}=0
$$

The constraints in Eq. (13) and Eq. (7) lead automatically to Eq. (10), which, together with Eq. (8), implies the expected ECQ, assigning to $h$ the correct electric charge, $Q_{h}=1$.

The second possibility allowed by the first term in Eq. (11) involves a scalar triplet, $\Delta \sim\left(\mathbf{1}, \mathbf{3}, Y_{\Delta}\right)$, composing the following Yukawa interaction with the lepton doublets:

$$
\mathcal{L}_{h}^{Y}=g_{i j} \overline{\left(L_{i_{L}}\right)^{c}} \Delta L_{j_{L}}+\text { H.c. }
$$

with $g_{i j}$ symmetric. This gives us the subsequent relations among the hypercharges,

$$
Y_{i_{L}}+Y_{j_{L}}+Y_{\Delta}=0
$$

which, together with Eq. (7) and Eq. (8), also result in ECQ.

Some comments are in order here. This scalar triplet is popular in the literature and has the following particle content:

$$
\Delta=\left(\begin{array}{ll}
\Delta^{0} & \frac{\Delta^{+}}{\sqrt{2}} \\
\frac{\Delta^{+}}{\sqrt{2}} & \Delta^{++}
\end{array}\right) .
$$

If we allow its neutral component, $\Delta^{0}$, to develop a vacuum expectation value (VEV), the (total) lepton number is spontaneously broken through its potential. The main consequences are that neutrinos acquire a mass at the tree level and a Majoron arises. However, such a Majoron is already excluded by experiments and it has to be avoided. To accomplish this, we assume that $\Delta^{0}$ does not develop a VEV, which is not a fine-tuning since this is an equally possible solution to the extremum equation that comes from demanding a minimum for the potential. In order to clearly see this, let us write down the potential involving the standard Higgs doublet, $H$, and the triplet, $\Delta$ :

$$
\begin{aligned}
V(\Phi, \Delta)= & \mu_{H}^{2} H^{\dagger} H+\lambda_{1}\left(H^{\dagger} H\right)^{2}+\mu_{\Delta}^{2} \operatorname{tr}\left(\Delta^{\dagger} \Delta\right) \\
& +\lambda_{2}\left[\operatorname{tr}\left(\Delta^{\dagger} \Delta\right)\right]^{2}+\lambda_{3} H^{\dagger} H \operatorname{tr}\left(\Delta^{\dagger} \Delta\right) \\
& +\lambda_{4} \operatorname{tr}\left(\Delta^{\dagger} \Delta \Delta^{\dagger} \Delta\right)+\lambda_{5}\left(H^{\dagger} \Delta^{\dagger} \Delta H\right) .
\end{aligned}
$$

From the minimum condition over this potential, we obtain

$$
\begin{aligned}
v_{H}\left[\mu_{H}^{2}+\lambda_{1} v_{H}^{2}+\frac{1}{2}\left(\lambda_{3}+\lambda_{5}\right) v_{\Delta}^{2}\right] & =0, \\
v_{\Delta}\left[\mu_{\Delta}^{2}+\lambda_{2} v_{\Delta}^{2}+\frac{1}{2}\left(\lambda_{3}+\lambda_{5}\right) v_{H}^{2}+\lambda_{4} v_{\Delta}^{2}\right] & =0 .
\end{aligned}
$$


According to the second relation above, one can promptly observe that $v_{\Delta}=0$ is an equally proper solution for the system above.

Since $\Delta^{0}$ does not develop a VEV, the total lepton number symmetry is kept intact and there will be no mixing among the particles of the triplet with those of the doublet. This means that the Goldstones in the theory (longitudinal components of $W^{ \pm}$and $Z$ ) come solely from the Higgs doublet, and the masses of the scalars that form the triplet, $M_{\Delta}$, depend only on $\mu_{\Delta}$ and $v_{H}$. We can safely assume $\mu_{\Delta} \simeq v_{H}$, which sets $M_{\Delta}$ in the electroweak scale, avoiding lower bounds over $\mu_{\Delta}$. This choice will be convenient when discussing the contribution of Eq. (14) to $(g-2)_{\mu}$ in Sec. IV.

The last possibility, which stems from the second term in Eq. (11), requires a scalar singlet, $k \sim\left(\mathbf{1}, \mathbf{1}, Y_{k}\right)$, interacting only with the charged lepton singlets,

$$
\mathcal{L}_{k}^{Y}=h_{i j} \overline{\left(l_{i_{R}}\right)^{c}} l_{j_{R}} k+\text { H.c. }
$$

where the coupling $h_{i j}$ is symmetric. The interaction above gives us the following relations among the hypercharges:

$$
Y_{i_{l}}+Y_{j_{l}}+Y_{k}=0
$$

which, together with Eq. (7) and Eq. (8), also implies the ECQ. Once having the ECQ, we see that the scalar involved in Eq. (19) carries two units of electric charge, $k \equiv k^{++}$.

\section{B. Lepton-quark interactions}

Another interesting possibility to include in this picture involves interactions like $\bar{\Psi}^{c} \Psi$, where one of the fermions is a lepton and the other a quark. However, the nature of the scalar interacting with these fermions is a little subtle, requiring that it carries both barion and lepton charges. Scalars like this are known in the literature as scalar leptoquarks. Any kind of interaction involving scalar leptoquarks leads to the ECQ and also gives contributions to $(g-2)_{\mu}$. The fermion representation content within the MSM is such that different kinds of scalar leptoquarks are allowed. In general, their interactions are classified by $F=0$ and $F=-2$, where $F$ is their assigned fermion number. However, the kind of bilinear fermion product we are interested in here leaves no room for leptoquark interactions with $F=0$, though they can also lead to ECQ [12]. Therefore, the remaining terms in the leptoquark Yukawa Lagrangian are $F=-2$ interactions [13],

$$
\begin{aligned}
\mathcal{L}_{F=-2}= & g_{L} \overline{\left(Q_{L}\right)^{c}} i \tau_{2} L_{L} \mathcal{S}_{0}^{L}+g_{R} \overline{\left(u_{R}\right)^{c}} l_{R} \mathcal{S}_{0}^{R} \\
& +\tilde{g}_{R} \overline{\left(d_{R}\right)^{c}} l_{R} \widetilde{\mathcal{S}}_{0}^{R}+g_{3 L} \overline{\left(Q_{L}\right)^{c}} i \tau_{2} \vec{\tau} L_{L} \overrightarrow{\mathcal{S}}_{1}^{L}+\text { H.c. }
\end{aligned}
$$

where $\mathcal{S}_{0}^{L}, \mathcal{S}_{0}^{R}$, and $\widetilde{\mathcal{S}}_{0}^{R}$ are singlets, while $\overrightarrow{\mathcal{S}}_{1}^{L}$ is a triplet. Despite the several interaction terms in Eq. (21), in what concerns ECQ just one of them would be sufficient. To be convinced of this, notice that any of the terms above connects the hypercharges of leptons with the hypercharges of quarks by

$$
Y_{\text {quarks }}+Y_{\text {leptons }}+Y_{\mathcal{S}}=0,
$$

which, together with Eq. (7) and Eq. (8), lead to the ECQ.

\section{THE $(g-2)_{\mu}$ DEVIATION}

The proposals studied in Sec. III are very appealing from a theoretical point of view, since they deal with simple modifications inside the as yet unknown scalar sector of the MSM, pointing to a solution for the ECQ problem. At first sight, some of them could only add to the list of those models already present in the literature. However, it is our main goal in this work to conciliate those scenarios with the recent $(g-2)_{\mu}$ measurement. Our intent is to show that except for the singlet extension, all the previous ECQ solutions can explain the posed deviation on $(g-2)_{\mu}$ with an adequate choice of parameters. This, by itself, would be a strong phenomenological motivation to suggest such economic modifications to the MSM.

Let us start by situating the $(g-2)_{\mu}$ problem, originating from a new measurement by the Brookhaven National Laboratory (BNL) experiment [11]. It indicates a deviation from the theoretical value of $2.6 \sigma$,

$$
a_{\mu}^{\exp }-a_{\mu}^{\mathrm{SM}}=(426 \pm 165) \times 10^{-11} .
$$

If this result persists [14], it implies an exciting window requiring new physics beyond the MSM. Among various scenarios proposed to account for the deviation, we restrict ourselves to those directly related to extensions in the scalar sector discussed in Sec. III $[13,15,16]$. As observed in Ref. [15], MSM extensions in the scalar sector have been almost neglected, mainly because Higgs contributions to $(g-2)_{\mu}$ can be significant only for light masses with usual values for Yukawa couplings $[17,18]$. Namely,

$$
f_{\mu \mu} \bar{\mu} \mu H
$$

gives the following contribution to $(g-2)_{\mu}[19]$ :

$$
a_{H}^{\mu}=\frac{f_{\mu \mu}^{2} m_{\mu}^{2}}{12 \pi^{2} m_{H}^{2}} .
$$

Here, $f_{\mu \mu}$ is the usual Yukawa coupling for the muon, and has the following form:

$$
f_{\mu \mu}=\frac{m_{\mu}}{v_{w}},
$$

where $m_{\mu}=0.105 \mathrm{GeV}$ is the muon mass and $v_{w}$ $=247 \mathrm{GeV}$ is the VEV of the scalar doublet in the MSM. These lead to

$$
f_{\mu \mu} \sim 10^{-3} \text {. }
$$

With this value for $f_{\mu \mu}$ and considering the Higgs mass of the order of hundreds of $\mathrm{GeV}, m_{H} \sim 10^{2} \mathrm{GeV}$, the standard Higgs contribution to $(g-2)_{\mu}$ is negligible,

$$
a_{H}^{\mu} \sim 10^{-13} \text {. }
$$


a)

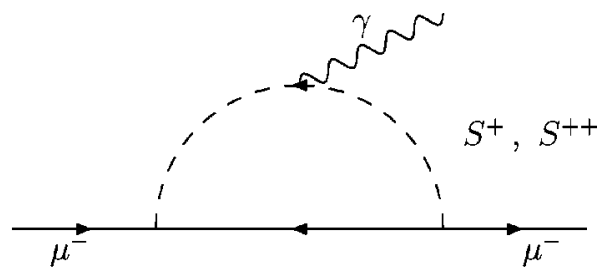

b)

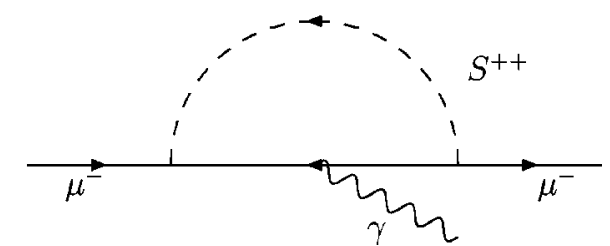

FIG. 1. General charged scalar $S$ contributions to the muon anomalous magnetic moment $(g-2)_{\mu}$.

Hence, if we wish to make minimal extensions mimicking this sector in order to explain the $(g-2)_{\mu}$ deviation, within a reasonable mass scale for the scalars, we have to impose some enhancement over the Yukawa couplings. This is, of course, an analysis which could be generalized to other scalar extensions, though in the case of leptoquarks we still can have small couplings for fairly large scalar masses [13]. The contribution to $(g-2)_{\mu}$ involving charged scalars is diagrammatically depicted in Fig. 1.

These pictures can be cast, respectively, in our particular case by the following expressions:

$$
\begin{aligned}
& \text { (a) } a_{\mu}^{S}=\frac{-C^{2} Q_{S}}{4 \pi^{2}} \int_{0}^{1} \frac{x^{3}-x^{2}}{x^{2}+(z-1) x+\frac{m_{f}^{2}}{m_{\mu}^{2}}(1-x)}, \\
& \text { (b) } a_{\mu}^{S}=\frac{C^{2}}{4 \pi^{2}} \int_{0}^{1} \frac{x^{2}-x^{3}}{x^{2}+z(1-x)},
\end{aligned}
$$

where $Q_{S}$ is the scalar charge, multiple of the positive electron charge $|e|>0, z$ is the ratio between the charged scalar and muon masses, $z=m_{S}^{2} / m_{\mu}^{2}$, and $C$ is a factor which involves the matrix coupling as well as a symmetry factor for each case (for $i=j$ this symmetry factor is 2 , and it is 1 otherwise). In the second formula above in Eq. (29), it is implicit that we are considering only a diagonal family interaction for the doubly charged scalar.

Let us first analyze the simplest case given by Eq. (12), where we added to the MSM only a singlet scalar interacting with fermions that, after assigning to it the correct electric charge, becomes

$$
\mathcal{L}_{h}^{Y}=f_{i j} \overline{\left(L_{i_{L}}\right)^{c}} L_{j_{L}} h^{+}+\text {H.c. }
$$

We recall that the coupling $f_{i j}$ is antisymmetric on the family indices $(i$ and $j$ ), and is roughly constrained if one considers $e-\mu-\tau$ universality [20], yielding

$$
\frac{f_{e \mu}^{2}}{m_{h}^{2}} \lesssim 2 \times 10^{-9} \mathrm{GeV}^{-2},
$$

which can be translated into $f_{e \mu} \lesssim 4 \times 10^{-3}$ for $m_{h}$ $\sim 100 \mathrm{GeV}$ (similar constraints can be imposed on $f_{e \tau}$ and $\left.f_{\mu \tau}\right)$.

With this value for the Yukawa coupling, we have $C$ $=0.5$ and $f_{i j}=2 \times 10^{-3}$, with $i=\mu$ and $j=e, \tau$ in the first expression of Eq. (29). Since the interaction involves a singly charged scalar, the fermion inside the loop has to be a neutrino, which is assumed massless in this work, so $m_{f}$ $=m_{\nu}=0$ in this case. However, in this regime we are not able to get any significant contribution to $(g-2)_{\mu}$ for reasonable scalar masses. We observe that this singly charged scalar can participate in more complex models, like the extended Zee model, where a second Higgs doublet, a second singly charged scalar, and a right-handed neutrino are added along with a new $\mathrm{U}(1)$ symmetry. These models aim to explain neutrino mass through radiative corrections and can lead to a substantial effect on $(g-2)_{\mu}[21]$ when the scalar mass is between $100<M_{S}<300 \mathrm{GeV}$. Although this alternative to the singlet singly charged scalar alone fits well in the scenario we have in mind, once the inclusion of such additional particles do not affect the achieved ECQ it is less appealing concerning its complexity.

The second case to be studied is the triplet one, with particle content given by Eq. (16). As we have seen in Sec. III, we can avoid phenomenological complications by taking the alternative solution for the VEV of this triplet to be zero. Still, we can assume its mass scale to be of the same order of the electroweak symmetry breaking scale, $M_{\Delta} \sim 200 \mathrm{GeV}$. In this range, the interaction term in Eq. (14) gives the appropriate contribution to $(g-2)_{\mu}$. In order to see this, observe that the interaction with singly and doubly charged scalars in Eq. (14) can be written explicitly as

$$
\mathcal{L}_{\Delta}^{\prime Y}=g_{i j}\left\{\left[\overline{\left(\nu_{i_{L}}\right)^{c}} l_{j_{L}}+\overline{\left(l_{i_{L}}\right)^{c}} \nu_{j_{L}}\right] \Delta^{+}+\overline{\left(l_{i_{L}}\right)^{c}} l_{j_{L}} \Delta^{++}\right\}+\text {H.c. }
$$

Here, $g_{i j}$ is symmetric on the family indices $i$ and $j$. Clearly what matters in solving the ECQ is the very property of such interactions to violate lepton flavor conservation, but in what concerns $(g-2)_{\mu}$, these violating terms are suppressed. This happens essentially because we are assuming scalars with mass on the electroweak symmetry breaking scale. For instance, consider only three of the flavor-changing processes: $\mu \rightarrow 3 e, \tau \rightarrow 3 \mu, 3 e$. The decay rate of a lepton, $l^{\prime}$, in three lighter leptons, $l$, allowed by the interaction in Eq. (32) has, in general, the following expression [20]:

$$
\Gamma\left(l^{\prime} \rightarrow 3 l\right) \simeq \frac{g_{l^{\prime} l}^{2} g_{l l}^{2}}{192 \pi^{3}} \frac{m_{l^{\prime}}^{5}}{m_{\Delta}^{4}} .
$$

The present experimental bounds on these flavors-changing processes are $B R(\mu \rightarrow 3 e) \leqslant 10^{-12}, B R(\tau \rightarrow 3 e, 3 \mu) \lesssim 10^{-6}$ [22]. These can be translated to the following constraints: $g_{e \mu} g_{e e} / m_{\Delta}^{2} \leq 10^{-11} \mathrm{GeV}^{-2}$ and $g_{e \tau} g_{e e} / m_{\Delta}^{2}, g_{\mu \tau} g_{\mu \mu} / m_{\Delta}^{2}$ $\$ 10^{-7} \mathrm{GeV}^{-2}$. If we have a scalar with mass, $m_{\Delta}$ $\simeq 10^{2} \mathrm{GeV}$, such constraints require $g_{e \mu} g_{e e} \lesssim 10^{-7}$ and $g_{e \tau} g_{e e}, g_{\mu \tau} g_{\mu \mu} \lesssim 10^{-3}$. Concerning the diagonal components, there is a lower bound to the product of $g_{e e}$ and $g_{\mu \mu}$ 
imposed by muonion-antimuoniun conversion: $g_{e e} g_{\mu \mu} / m_{\Delta}^{2}$ $>10^{-8} \mathrm{GeV}^{-2}$ [23]. For $m_{\Delta} \simeq 10^{2} \mathrm{GeV}$, we have $g_{e e} g_{\mu \mu}$ $>10^{-4}$. Along with this, the only upper bounds come from $(g-2)_{e}$ and the Bhabha scattering process. From these, the last is the most stringent [24]: $g_{e e}^{2} / m_{\Delta}^{2}<10^{-6} \mathrm{GeV}^{-2}$, which requires, in our case, $g_{e e}<10^{-1}$. There is no experimental constraint on $g_{\mu \mu}$, except for the recent $(g-2)_{\mu}$ deviation, which we are going to expose below.

Since we are interested in enhanced diagonal Yukawa couplings, it is clear that the off-diagonal ones are suppressed by, at least, three orders of magnitude. In this context, we can safely assume $g_{\mu \mu} \simeq 1$ and compute $(g-2)_{\mu}$ to set the suitable mass range for the scalar, $\Delta$, which would render agreement between theory and experiment. In the triplet case, both expressions in Eq. (29) have to be employed. The first of these expressions accounts for a $\Delta^{+}$as well as a $\Delta^{++}$exchange, while the second only involves $\Delta^{++}$. The singly charged scalar contribution is similar to the singlet case, except for the Yukawa coupling, which is diagonal in this case and of the order of unity, leading to $C=0.5$ for this computation. For the doubly charged scalar exchange, $C$ $=1$ at both expressions in Eq. (29). In order to explain the observed deviation in $(g-2)_{\mu}$, we have to keep the triplet mass between $200 \leqq m_{\Delta} \lesssim 300 \mathrm{GeV}$, which makes it an attractive proposal. Not only does it solve simultaneously the ECQ puzzle, but for it forces a mass range which could be easily prompted in the next generation of accelerators.

We consider now the doubly charged scalar singlet, Eq. (19). This extension was already studied in Ref. [15], wherein it was verified that such a scalar is relevant for the $(g-2)_{\mu}$ problem only if its mass is around $200 \mathrm{GeV}$. This is not surprising, since the triplet case studied above only differs from this case by a singly charged scalar contribution, which is not as important as the doubly charged one because the former is almost one order of magnitude lower than the last. Hence, this scenario is an equally good candidate to be the solution for both the ECQ and $(g-2)_{\mu}$ problems.

Finally, we can discuss the role of scalar letpoquark interactions, as given by Eq. (21), in the context of $(g-2)_{\mu}$, assessed in Refs. [13,16]. In Ref. [16], the mixing among generations was allowed, which could lead to problems concerning flavor-changing neutral currents. This was avoided in Ref. [13] by assuming there was no such generation mixing and the author obtained that the only important effect on $(g-2)_{\mu}$ occurs when the leptoquark has both left- and righthanded couplings to fermions, although leptoquarks coupling with only one type of handedness can coexist harmlessly. We will adopt this approach here. In our case, these leptoquarks are identified in the Yukawa Lagrangian, Eq. (21), as $\mathcal{S}_{0}^{L, R}$. The bounds put by $(g-2)_{\mu}$ over their masses are, 0.7 $<M_{\mathcal{S}}<2.0 \mathrm{TeV}$, and were obtained considering couplings of electromagnetic size [13].

In this context, it is still possible to assume first-second generation universality or not. The first possibility puts additional constraints on the allowed leptoquark interaction through $\eta_{C C}[13] .^{2}$ To avoid this, the singlet scalar leptoquark has to be accompanied by the triplet, which does not significantly contribute to $(g-2)_{\mu}$ while modifying $\eta_{C C}$ in the right direction such as to compensate for the effect of the singlet. The second possibility alone is enough to circumvent this complication since it leads to the desired effect on $(g$ $-2)_{\mu}$ without requiring the presence of other leptoquarks. As the coexistence of additional leptoquarks does not jeopardize our picture, both possibilities are welcome, though the second is more economic.

\section{CONCLUSIONS}

In this work, we have suggested small extensions of the MSM by augmenting the scalar sector on minimal portions, aiming to explain both the long-standing problem of electric charge quantization and the measured deviation of the muon anomalous magnetic moment, recently reported by the BNL experiment. The scalars suggested here are not usual ones, however, since they must couple to bilinear fermionic products, $\bar{\Psi}^{c} \Psi$, and consequently carry two units of lepton number. The purpose is to oblige the new Yukawa interactions to explicitly induce family mixing, eliminating the hidden symmetries which impede the realization of ECQ inside the MSM. Notice that such extensions do not interfere with other symmetries of the MSM, keeping them intact.

Among the proposed scalars, there are singlets, doubly and singly charged, a single triplet, and also leptoquarks, disposed in simple configurations. All of them are fair candidates to simultaneously achieve ECQ and an explanation for the $(g-2)_{\mu}$, except for the singly charged singlet, which accounts for ECQ but is insufficient to properly solve the $(g-2)_{\mu}$ discrepancy. This singlet could be inserted in a more complex configuration in order to accomplish this double task, although we would rather stick to plain extensions of MSM. It is interesting to remark that the scalar masses rendered by this study are close to the experimental reach of the next generation of accelerators.

In summary, we successfully managed to relate some solutions for the ECQ with the theory-experiment deviation on the muon anomalous magnetic moment. The whole picture impels us to suggest that the agreement between experiment and theory leads to the next step, which in this situation is a modest expansion of the MSM scalar sector.

\section{ACKNOWLEDGMENTS}

This work was supported by Fundâão de Amparo à Pesquisa do Estado de São Paulo (FAPESP).

\footnotetext{
${ }^{2} \eta_{C C}=\eta_{L L}^{e d}-\eta_{L L}^{e u}=(0.051 \pm 0.037) \mathrm{TeV}$, where $\eta_{\alpha \beta}^{l q}$ is a contact parameter, with $\alpha$ and $\beta$ denoting the chirality of the lepton $(l)$ and the quark $(q)$, used to parametrize the four-fermion effective interaction that appears in a regime where the mass of the leptoquarks is larger than the energy scale involved in the experiment.
} 
[1] R. Foot, G.C. Joshi, H. Lew, and R.R. Volkas, Mod. Phys. Lett. A 5, 2721 (1990); R. Foot, H. Lew, and R.R. Volkas, J. Phys. G 19, 269 (1993).

[2] K.S. Babu and R.N. Mohapatra, Phys. Rev. D 41, 271 (1990).

[3] J. Sladkowski and M. Zralek, Phys. Rev. D 45, 1701 (1992).

[4] K.S. Babu and R.N. Mohapatra, Phys. Rev. Lett. 63, 938 (1989).

[5] C.Q. Geng and R.E. Marshak, Phys. Rev. D 39, 693 (1989); C.Q. Geng, ibid. 41, 1292 (1990); E. Golowich and P.B. Pal, ibid. 41, 3537 (1990); S. Rudaz, ibid. 41, 2619 (1990).

[6] M. Nowakowskia and A. Pilaftsis, Phys. Rev. D 48, 259 (1993).

[7] K.S. Babu and R.N. Mohapatra, Phys. Rev. D 42, 3866 (1990).

[8] C.A. de S. Pires and O.P. Ravinez, Phys. Rev. D 58, 035008 (1998); C.A. de S. Pires, ibid. 60, 075013 (1999).

[9] S. Adler, Phys. Rev. 177, 2426 (1969); J.S. Bell and R. Jackiw, Nuovo Cimento A 60, 49 (1969).

[10] R. Foot and R.R. Volkas, Phys. Rev. D 59, 097301 (1999).

[11] H.N. Brown et al., Phys. Rev. Lett. 86, 2227 (2001).

[12] J.P. Bowes, R. Foot, and R.R. Volkas, Phys. Rev. D 54, 6936 (1996).

[13] K. Cheung, Phys. Rev. D 64, 033001 (2001).

[14] F.J. Ynduráin, hep-ph/0102312.

[15] C.A. de S. Pires and P.S. Rodrigues da Silva, Phys. Rev. D 64, 117701 (2001).

[16] U. Mahanta, Eur. Phys. J. C 21, 171 (2001); D. Chakraverty, D. Choudhury, and A. Datta, Phys. Lett. B 506, 103 (2001).

[17] A. Dedes and H.E. Haber, J. High Energy Phys. 05, 006 (2001); M. Krawczyk, hep-ph/0103223.

[18] For some recent works on the subject with other scalars, see A. Czarnecki and W.J. Marciano, Phys. Rev. D 64, 013014 (2001); L. Everett, G.L. Lane, S. Rigolin, and L.T. Wang,
Phys. Rev. Lett. 86, 3484 (2001); J.L. Feng and K.T. Matchev, ibid. 86, 3480 (2001); E.A. Baltz and P. Gondolo, ibid. 86, 5004 (2001); U. Chattopadhyay and P. Nath, ibid. 86, 5854 (2001); S. Komine, T. Moroi, and M. Yamaguchi, Phys. Lett. B 506, 93 (2001); U. Mahanta, ibid. 511, 235 (2001); T.W. Kephart and H. Pas, hep-ph/0102243; E. Ma and M. Raidal, Phys. Rev. Lett. 87, 011802 (2001); Z.H. Xiong and J.M. Yang, Phys. Lett. B 508, 295 (2001); A. Dedes and H.E. Haber, [17]; hep-ph/0105014; Z. Xing, Phys. Rev. D 64, 017304 (2001); J. Hisano and K. Tobe, Phys. Lett. B 510, 197 (2001); J. Ellis, D.V. Nanopoulos, and K.A. Olive, ibid. 508, 65 (2001); R. Arnowitt, B. Dutta, and Y. Santoso, ibid. 505, 177 (2001); T. Kobayashi and H. Terao, Phys. Rev. D 64, 075003 (2001); M.B. Einhorn and J. Wudka, Phys. Rev. Lett. 87, 071805 (2001); R.A. Diaz, R. Martinez, and J.A. Rodriguez, Phys. Rev. D 64, 033004 (2001); S.K. Kang and K.Y. Lee, Phys. Lett. B 521, 61 (2001); S.P. Martin and J.D. Wells, Phys. Rev. D 64, 035003 (2001); S. Rajpoot, hep-ph/0103069; H. Baer, C. Balazs, J. Ferrandis, and X. Tata, Phys. Rev. D 64, 035004 (2001); K. Choi, K. Hwang, S.K. Kang, K.Y. Lee, and W.Y. Wan, ibid. 64, 055001 (2001); S. Baek, N.G. Deshpande, X.G. He, and P. Ko, ibid. 64, 055006 (2001); A. Arhrib and S. Baek, ibid. 65, 075002 (2002).

[19] S.R. Moore, K. Whisnant, and B.L. Young, Phys. Rev. D 31, 105 (1985).

[20] K. Babu, Phys. Lett. B 203, 132 (1988).

[21] D.A. Dicus, H.J. He, and J.N. Ng, Phys. Rev. Lett. 87, 111803 (2001).

[22] Particle Data Group, D. Groom et al., Eur. Phys. J. C 15, 1 (2000).

[23] R.N. Mohapatra and P.B. Pal, Massive Neutrinos in Physics and Astrophysics, 2nd ed. (World Scientific, Singapore, 1998).

[24] M.L. Swartz, Phys. Rev. D 40, 1521 (1989). 